Original

\title{
Simulation Study of Competition between Two Types of Microorganisms with Antagonistic Relationships in a Completely Mixed Reactor
}

\author{
SATOSHI SODA, MICHIHIKO IKE, AND MASANORI FUJITA* \\ Department of Environmental Engineering, Osaka University, \\ 2-1, Yamada-oka, Suita, Osaka 565-0871, Japan
}

Received 7 November 1998/Accepted 9 February 1999

\begin{abstract}
A simple dynamic model of competition between two types of microorganisms, one of which produces an inhibitory substance in an ideal chemostat, was developed to study the survival mechanism of an exogenous microbial strain introduced into an ecosystem possessing antagonistic activity against it. Numerical simulations of the model were carried out in 12 cases depending on the disposition of the growth rate curves of the two types of microorganisms and on the sensitivity of the microorganisms against the inhibitory substance. From the results, it was demonstrated that production of an inhibitory substance gives its producer a chance to exclude the competitor under conditions in which the producer would be otherwise excluded by the competitor. It was also found that production of a substance which is inhibitory not only against the competitor but also against the producer itself (autoinhibitory) allows the two microbial strains to coexist under conditions in which the competitor would otherwise be excluded. It was suggested that production of some inhibitory substances and tolerance to such substances are desirable characteristics for exogenous microorganisms to survive in microbial ecosystems, and a large inoculum density of exogenous microorganisms has a positive effect on their survival rate in some cases.
\end{abstract}

Key words : Antagonism/Competition/Modeling/Survival.

\section{INTRODUCTION}

There has been much concern regarding the intentional release to natural ecosystems of exogenous microorganisms with desirable capabilities for bioremediation of polluted environments (bioaugmentation). However, many researchers have reported that such exogenous microorganisms, after being introduced, drastically decreased in a variety of environments (Barcina et al., 1997; Gurijala and Alexander, 1990). In our previous report, the drastic decrease of exogenous bacterial populations was also observed in fresh water, sea water, activated sludge, and soil microcosms (Soda et al., 1998). The

${ }^{*}$ Corresponding author. Tel: +81-6-6879-7672, Fax : +816-6879-7675. experimental results indicated that the interaction with indigenous bacteria, especially in the form of antagonism, was the most influential lethal factor against the exogenous bacterial populations, and approximately $1.0-3.5 \%$ of the isolated indigenous bacterial strains from the microcosms showed antagonistic activity against the exogenous bacteria. Several other researchers have also reported that antagonistic bacteria which produce inhibitory substances are widely distributed and that many inhibitory substances are present in microbial ecosystems (Dopazo et al., 1988; Gauthier and Flatas, 1976; Klein and Alexander, 1986; Lemos et al., 1985; Nair and Shimidu, 1987; Rosenfeld and ZoBell, 1947; Tang and Alexander, 1987; Zaidi et al., 1989).

Although natural microbial habitats involve heterogeneity, the competition of microorganisms in a 
homogenous environment has been the subject of many studies (Aris and Humphrey, 1977; Fredrickson and Stephanpoulos, 1981; Lenas and Pavlou, 1995; Lenski, 1986; Schoener, 1976; Vance, 1986). For successful inoculation of exogenous microorganisms into microbial ecosystems where indigenous antagonistic microorganisms exist, it is also necessary to understand the ecological role of antagonism in a homogenous ecosystems. De Freitas and Fredrickson (1978) studied the ecological role of the inhibitory substrate which is inhibitory against the growth of microorganisms using a simple mathematical model. They concluded that the production of inhibitory substrates by microorganisms may be a relevant factor in maintaining the species diversity of microbial ecosystems. In this present study, antagonistic competition between two types of microorganisms in a completely mixed reactor was simulated in 12 cases depending on the disposition of the growth rate curves and on the microbial sensitivity to the inhibitory substance. Based on the simulations, a few possible strategies for effective bioaugmentation will be discussed.

\section{MODEL DEVELOPMENT}

\section{Governing equations}

First, it was assumed that microorganism types 1 and 2 compete for a common substrate in an idealized completely mixed reactor. In addition, it was assumed that the production rate of the inhibitory substance was proportional to the growth rate of its producer, and that neither microbial strain consumes the inhibitory substance. The relationships assumed between the two strains are illustrated in Fig. 1. The mass balances in the reactor are expressed by the following equations:

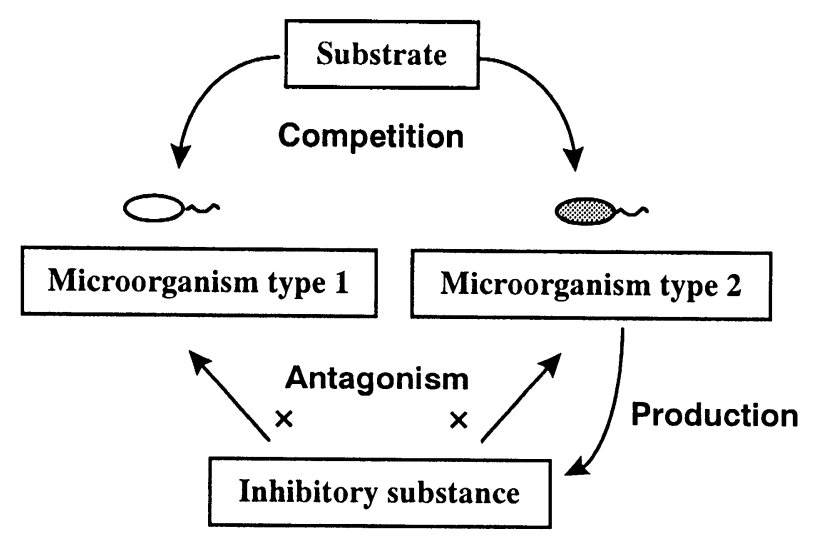

FIG. 1 . Antagonistic relationship between microorganism types 1 and 2.

$$
\begin{aligned}
& \frac{d X_{i}}{d t}=\left(\mu_{i}-D\right) X_{i} \\
& \frac{d S}{d t}=\left(S_{f}-S\right) D-\sum \frac{\mu_{i} X_{i}}{Y_{i}} \\
& \frac{d l}{d t}=-I D+\sum p_{i} \mu_{i} X_{i}
\end{aligned}
$$

where $X, S, I$ are the biomass, concentration of the substrate, and concentration of the inhibitory substance, respectively. $S_{f}$ is the substrate concentration of the influent into the reactor, while $D$ is its dilution rate. $\mu, Y$, and $p$ are the growth rate constant, yield constant, and production constant of the inhibitory substance, respectively. $i$ is the suffix for distinguishing each microbial strain. The growth of the microorganisms is expressed by the following equation which is a typical modified Monod equation for product inhibition (Han and Levenspiel, 1988):

$$
\mu_{i}=\frac{\mu_{m i} S}{K_{S i}+S}\left(1-\frac{l}{C_{l i}}\right)
$$

where $\mu_{\mathrm{m}}, K_{s}$, and $C_{l}$ are the maximum growth rate constant, half saturation constant, and inhibition constant for the ith microorganism, respectively. In view of the inhibitory mechanism, there are two types of inhibitory substances: one shows inhibition against the microbial growth (Lemos et al., 1985; Nair and Simidu, 1987; Rosenfeld and ZoBell, 1947) and the other is lethal to microorganisms (Jemsen, et al., 1993; Tang and Alexander, 1987). In this model, the parameter $C_{l}$ indicates the concentration of the inhibitory substance at which the growth of the microorganisms is completely inhibited. When the concentration of the inhibitory substance is higher than the $C_{\text {, value, }}$ the growth rate constant becomes below zero, leading to a decay of the microorganisms. A smaller $C_{\text {, value }}$ refers to a stronger inhibitory effect.

\section{Parameters}

For simulation studies, it was assumed that only microorganism type 2 produces an inhibitory substance, i. e., microorganism type 1 is assumed to be the exogenous strain and type 2 the indigenous strain in the

TABLE 1. Parameters of the microorganisms concerning the inhibitory substance in cases 1-3.

\begin{tabular}{ccccc}
\hline Case & $C_{11}(\mathrm{mg} / \mathrm{I})$ & $C_{12}(\mathrm{mg} / \mathrm{I})$ & $p_{1}(-)$ & $p_{2}(-)$ \\
\hline 1 & 1.0 & $\infty$ & 0.0 & 0.1 \\
2 & 1.0 & 1.0 & 0.0 & 0.1 \\
3 & 1.0 & 0.5 & 0.0 & 0.1 \\
\hline
\end{tabular}



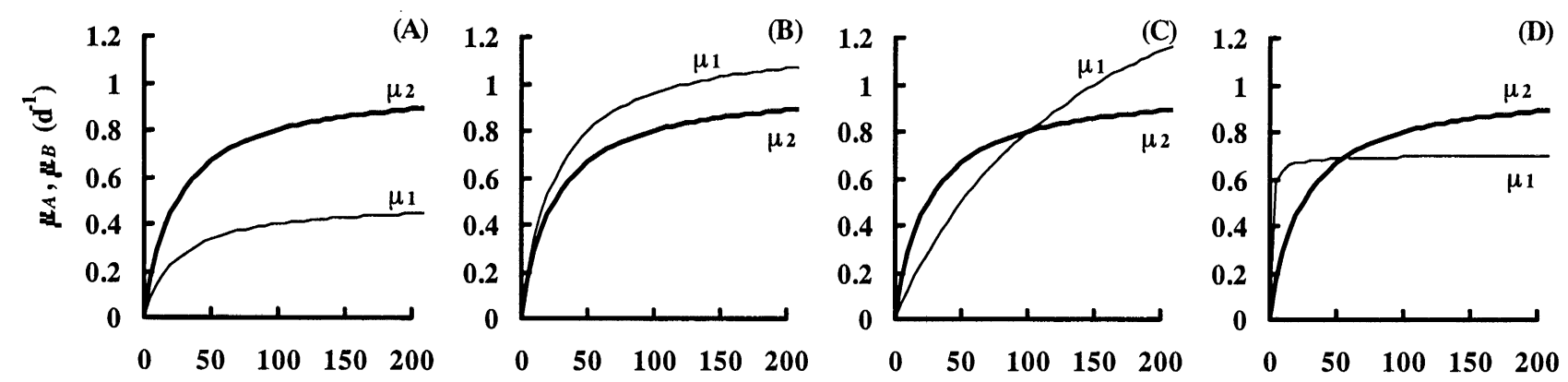

\section{$S(\mathrm{mg} / \mathrm{l})$}

FIG. 2. Disposition of growth rate curves of the microorganisms when their growth is not affected by the inhibitory substance. (A) - (D) correspond to cases A - D in Table 2.

case of practical bioaugmentation. Some inhibitory substances can also show inhibition against their producer (autoinhibition) (Lemos et al., 1985; Nair and Shimidu, 1987); therefore, three cases, 1-3, were considered as shown in Table 1 . In case 1, microorganism type 2 is not affected by the inhibitory substance. In case 2, microorganism type 2 is as sensitive as type 1 to the inhibitory substance. In case 3 , microorganism type 2 is more sensitive to the inhibitory substance than type 1 . The survival of microorganisms also considerably depends on their disposition of growth rate curves (Aris and Humphery, 1977). Therefore, 4 disposition cases, A-D, were assumed as shown in Table 2. The disposition of the growth rate curves when their growth is not affected by the inhibitory substance are shown in Fig. $2\left(C_{11}=C_{12}=\infty\right)$. In case $A$, microorganism type 2 grows faster than type 1 at any substrate concentration. In case B, microorganism type 1 grows faster than type 2 at any substrate concentration. In case $\mathrm{C}$, microorganism type 1 grows faster at high substrate concentrations, while in case $D$, microorganism type 2 grows faster at high substrate concentrations. In total, 12 cases, combination of cases 1-3 and cases A-D, were assumed. All simulations were carried out using the 4th order Runge-Kutta method provided by the software ISIM for Windows (Snape et al., 1995).

\section{RESULTS}

\section{Operational diagrams}

The nature of the solutions of the equations depends on the microbial parameters in each case and on the operational parameters, $D$ and $S_{f}$. This dependence is summarized in an operational diagram. An operational diagram is a $D-S_{f}$ plane where various steady-state solution of the model equations exist and are stable with respect to small perturbations. The diagrams in the 12 cases consist of 5 regions, I-V
TABLE 2. Parameters of the microorganisms concerning the disposition of the growth rate curves in cases A-D.

\begin{tabular}{ccccc}
\hline Case & $\mu_{m 1}\left(\mathrm{~d}^{-1}\right)$ & $K_{s 1}(\mathrm{mg} / \mathrm{l})$ & $\mu_{m 2}\left(\mathrm{~d}^{-1}\right)$ & $K_{s 2}(\mathrm{mg} / \mathrm{l})$ \\
\hline A & 0.5 & 25 & & \\
B & 1.2 & 25 & 1.0 & 25 \\
C & 2 & 150 & & \\
D & 0.7 & 1.0 & & \\
\hline
\end{tabular}

(Fig. 3). In region I, both microbial strains are washed out from the reactor. In regions II, microorganism type 1 is washed out but type 2 survives in the reactor. Conversely, in region III, microorganism type 2 is washed out but type 1 survives in the reactor. In region IV, microorganism type 1 or 2 is washed out depending on the initial conditions of $S, I, X_{1}$, and $X_{2}$ (multi-steady-state). Interestingly, in region $\mathrm{V}$, the stable steady-state is the coexistence of microorganism types 1 and 2.

Region IV is found in cases (1-B), (1-D), (2-D), and (3-D) and region $\mathrm{V}$ in cases (2-C), (3-A), and (3-C), and these two regions are not found when the typical Monod equation is applied to simulations of competition of two types of microorganisms for a single substrate. Without antagonistic relationships, microorganism types 1 and 2 cannot survive in regions $\mathrm{V}$ and IV, respectively. Decrease in $C_{12}$ value makes region IV smaller as shown in cases $B$ and $D$, and does region $\mathrm{V}$ larger as shown in cases $\mathrm{A}$ and $\mathrm{C}$.

\section{Multi-steady-state in region IV}

Trajectories in $X_{1}, X_{2}, S$ space in case (2-D) at 0.5 $\mathrm{d}^{-1}$ of $D$ and $100 \mathrm{mg} / /$ of $S_{f}$ are shown in Fig. 4. The initial condition of the / value was set at (A) $0.0 \mathrm{mg} / /$ and (B) $2.0 \mathrm{mg} / /$. Under the operational conditions, there are two stable cases of steady-state, $P\left(X_{1}, X_{2}\right.$, $S, I)$ and $Q\left(X_{1}, X_{2}, S, I\right)$, which are $(48,0.0,2.5,0.0)$ and $(0.0,3.6,92,0.36)$, respectively. The higher initial concentration of the inhibitory substance, the 


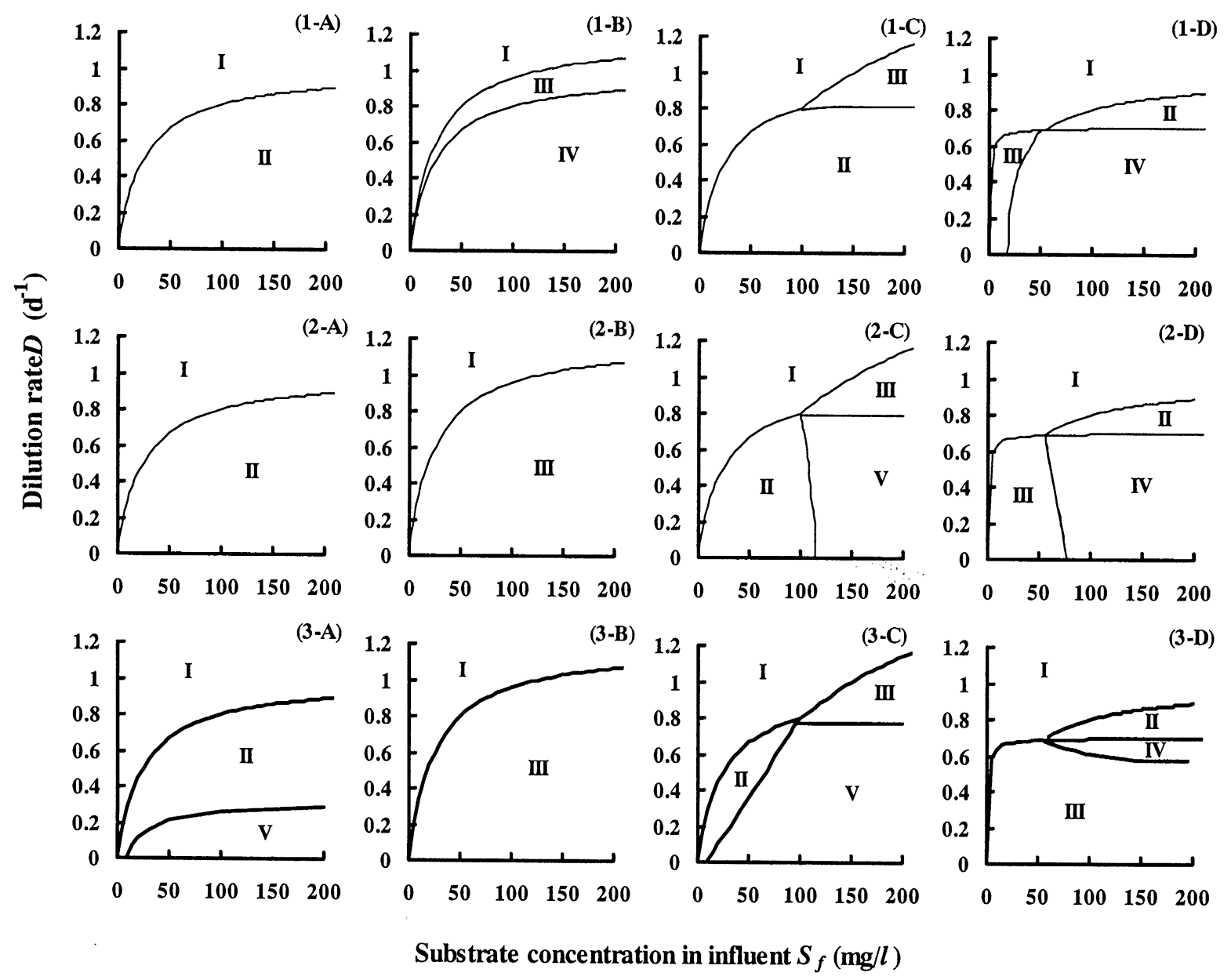

FIG. 3. Operational diagrams in the 12 cases. In region I, both types of microorganisms are washed out. In region II, microorganism type 1 is washed out. In region III, microorganism type 2 is washed out. In region IV, microorganism type 1 or 2 is washed out depending on the initial conditions. In region $\mathrm{V}$, microorganisms coexist. (1-A) - (3-D) indicate combination of cases 1-3 in Table 1 and cases A- D in Table 2.
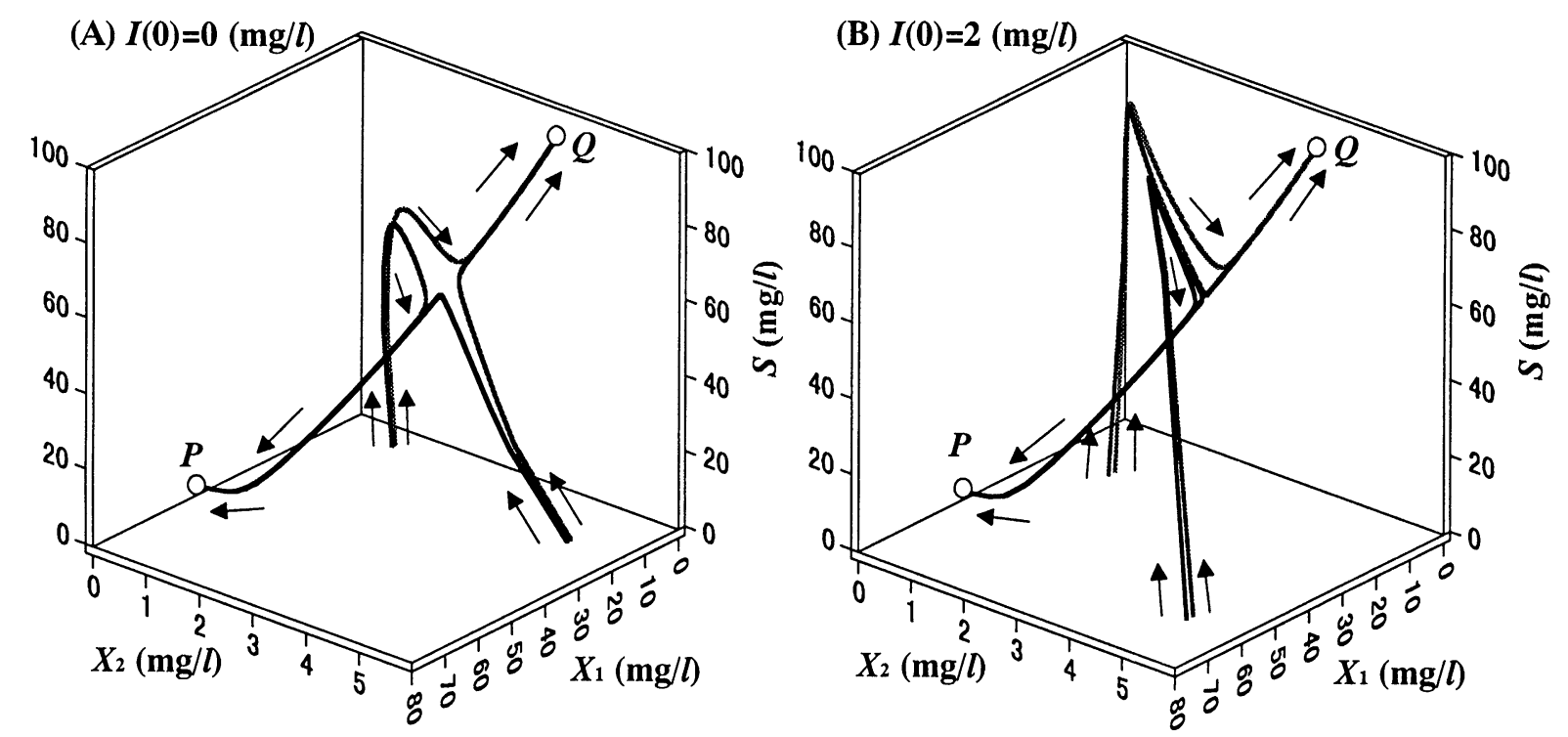

FIG. 4. Trajectories in $X_{1}, X_{2}, S$ space in case (2-D) with (A) $0 \mathrm{mg} / /$ and (B) $2.0 \mathrm{mg} / /$ of the initial / value. Two cases of stable steady-state, $P$ and $Q$, are also shown. 
larger initial biomass is needed for microorganism type 1 to survive in the reactor. $X_{1}$ and $S$ at the stable steady-point $P$ are expressed by the following equations:

$$
\begin{aligned}
& S=\frac{D K_{s 1}}{\mu_{m i}-D} \\
& X_{1}=Y_{1}\left(S_{f}-S\right)
\end{aligned}
$$

The stable steady-state in region III is also expressed by the equations (5) and (6). $X_{2}, S$, and / at the stable steady-point $Q$ are expressed by the following equations:

$$
\begin{aligned}
& S=\frac{-b+\sqrt{b^{2}+4 a c}}{2 a} \\
& X_{2}=Y_{2}\left(S_{f}-S\right) \\
& I=p_{2} Y_{2}\left(S_{f}-S\right)
\end{aligned}
$$

where $a=\frac{\mu_{m 2} p_{2} Y_{2}}{C_{12}}, b=\mu_{m 2}-D-\frac{\mu_{m 2} p_{2} Y_{2} S_{f}}{C_{12}}, c$ $=-K_{s_{2}} D$. The stable steady-state condition in region II is also expressed by the equations (7) - (9).

\section{Coexistence of the microorganisms in region $\mathrm{V}$}

Figure 5 shows the effect of the dilution rate and the
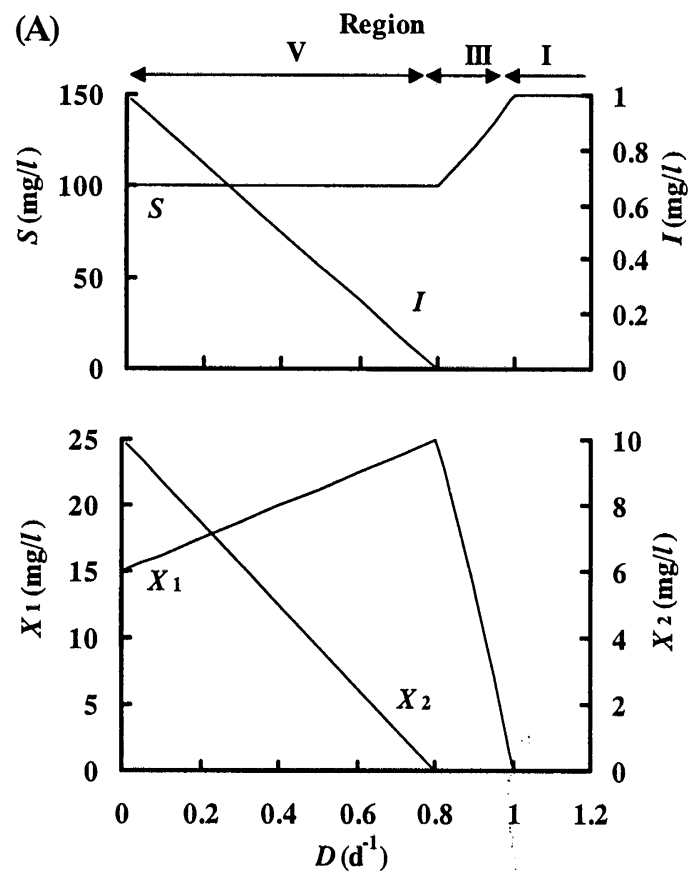

substrate concentration in the influent on the stable steady-state in case (2-C). The autoinhibition acts to make the growth rate of microorganism type 2 partially self-limiting, and allows the coexistence of $\mathrm{mi}$ croorganism types 1 and 2 . In region $\mathrm{V}$, the stable steady-state is expressed by the following equations:

$$
\begin{aligned}
& S=\frac{D\left(C_{12} \mu_{m 1} K_{s 2}-C_{11} \mu_{m 2} K_{s 1}\right)}{\mu_{m 1} \mu_{m 2}\left(C_{l 2}-C_{l 1}\right)+D\left(\mu_{m 2} C_{l 1}-\mu_{m 1} C_{11}\right)} \\
& I=\frac{D\left(K_{s 1}-K_{s 2}\right)+\mu_{m 1} K_{s 2}-\mu_{m 2} K_{s 1}}{\frac{\mu_{m 1} K_{s 2}}{C_{l 1}}-\frac{\mu_{m 2} K_{s 1}}{C_{l 2}}} \\
& X_{1}=\frac{p_{2} Y_{1} Y_{2}\left(S_{t}-S\right)-Y_{1} I}{p_{2} Y_{2}-p_{1} Y_{1}} \\
& X_{2}=\frac{p_{1} Y_{1} Y_{2}\left(S_{t}-S\right)-Y_{2} I}{p_{1} Y_{1}-p_{2} Y_{2}}
\end{aligned}
$$

In region $\mathrm{V}$ of this case, the $S$ value is independent of the $D$ value because $C_{11}$ equals $C_{12}$.

\section{DISCUSSION}

In spite of several successful small-scale bioaugmentation tests, the inoculation of specialized microorganisms to natural environments to enhance the removal of pollutants has not been widely applied.
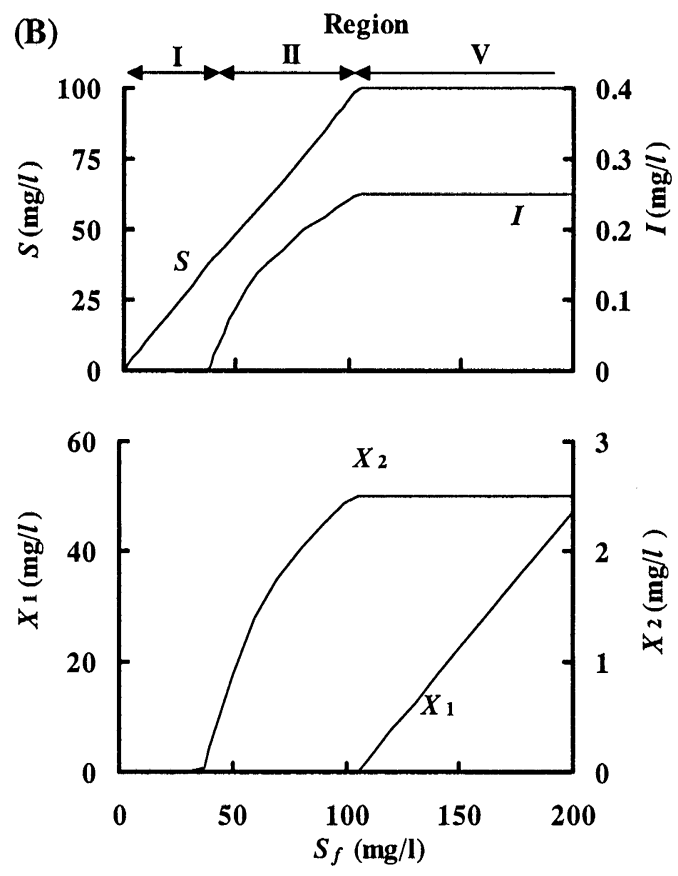

FIG.5. Effect of operational parameters on the stable steady-state in case (2-C). The effect of the dilution rate at $150 \mathrm{mg} / \mathrm{l}$ of $S_{f}$ value (A) and effect of the substrate concentration in the influent at $0.6 \mathrm{~d}^{-1}$ of the $D$ value (B). 
This may be because the behavior of exogenous microorganisms in microbial ecosystems has not been fully studied and is less predictable and controllable than the physical or chemical destruction of pollutants. Competition between two types of microorganisms for a single substrate never allows them to coexist if it depends on only their ability to utilize the substrate (Aris and Humphrey, 1977). In contrast, competition with antagonistic relationships can lead to various cases of stable state, coexistence and selection depending on not only the substrate concentration but also on the initial biomass. Results presented here supports our previous report that indigenous microorganisms with antagonistic activity affect the behavior of exogenous microorganisms inoculated into microbial ecosystems (Soda et al., 1998).

Needless to say, the small $K_{s}$ value and the large $\mu_{m}$ value are desirable parameters for microorganisms to be able to survive in a mixed microbial ecosystem. However, the simulation study here indicated that the presence of an antagonistic relationship can be also an important factor which may considerably affect their survival. The simulation results qualitatively correspond with the conclusion of De Freitas and Fredrickson (1978), that the production of a substance which is inhibitory against the competitor gives its producer a chance to exclude its competitor under conditions where the producer would be otherwise excluded by the competitor. The initial condition is demonstrated to be very important for survival of the microorganisms with some antagonistic relationship as shown in Fig. 4. A large inoculum density of exogenous microorganisms may be effective for bioaugmentation in cases (1-B), (1-D), (2-D), and (3D). This result may support our previous observation that a large inoculum size was needed for genetically engineered bacteria to survive in activated sludge (Fujita et al., 1994).

Based on the results shown in Fig. 3, breeding and/ or selection of exogenous microorganisms tolerant to some inhibitory substances may be a possible strategy to enhance their chances of survival. Enhanced survival of genetically engineered microorganisms which are resistant to some antibiotics in soil microcosms have already been observed by simultaneous addition of the antibiotics (da Gloria et al., 1995). Another strategy is breeding and/or selection of exogenous microorganisms which produce inhibitory substances. Genetically engineered microorganisms with such ability have already been constructed as a suicide system to control the survival of the microorganisms (Jemsen et al., 1993; Ramos et al., 1994). However, this strategy will need to be more thoroughly examined and discussed in terms of the problem of biohazards. Actually, an undesirable result of the bioaugmentation to a soil microcosm has been also reported: a toxic metabolite produced by a genetically engineered bacterium depressed fungal propagules and respiration of the soil microbiota (Short et al. 1991).

From the simulations, it was also confirmed that production of a substance which is not only inhibitory against its competitor but also autoinhibitory allows coexistence under wide conditions in which the competitor would otherwise be excluded. Existence of predatory microorganisms (Lotka, 1920), spatially heterogeneous habitats such as soil particles and biofilms (Wanner and Gujer, 1986), and periodic variation of operational conditions (Lenas and Pavlou, 1995) have been suggested as reasons for the coexistence of microorganisms. However, the coexistence of two types of microorganisms in a completely mixed reactor expressed by the typical Monod equation can be observed only at the very point where the dilution rate exactly equals the crossing point of the two growth curves (Aris and Humphrey, 1977). This study suggests that the antagonism may be one of the possible reasons for the coexistence of microorganisms and should aid in the development of an appropriate mathematical model describing the survival mechanism of exogenous microorganisms in microbial ecosystems.

\section{NOMENCLATURE}

$C_{l} \quad$ : Inhibition constant $(\mathrm{mg} / \mathrm{I})$

$D$ : Dilution rate $\left(\mathrm{d}^{-1}\right)$

$K_{s}:$ Half saturation constant $(\mathrm{mg} / \mathrm{I})$

I : Inhibitory substance concentration ( $\mathrm{mg} / \mathrm{I})$

$p$ : Production rate of inhibitory substance (-)

$S$ : Substrate concentration $(\mathrm{mg} / \mathrm{I})$

$S_{f}$ : Substrate concentration in influent $(\mathrm{mg} / \mathrm{I})$

$X$ : Biomass (mg//)

$Y$ : Yield constant (-)

$\mu \quad$ : Growth rate constant $\left(\mathrm{d}^{-1}\right)$

$\mu_{m}$ : Maximum growth rate constant $\left(\mathrm{d}^{-1}\right)$

\section{Subscripts}

1: Microorganism type 1

2 : Microorganism type 2

\section{REFERENCES}

Aris, R., and Humphrey, A. E. (1977) Dynamics of a chemostat in which two organisms compete for a common substrate. Biotechnol. Bioeng., 19, 13751386. 
Barcina, I., Lebaron, P., and Vives-Rego, J. (1997) Survival of allochthonous bacteria in aquatic system: a biological approach. FEMS Microbiol. Ecol., 23, 1-9.

da Gloria, R., de Oliveira, B., Wolters, A. C., and van Elas, J. D. (1995) Effects of antibiotics in soil on the population dynamics of transposon Tn5 carrying Pseudomonas fluorescens. Plant and Soil, 175, 323-333.

De Freitas, M. J., and Fredrickson, A. G. (1978) Inhibition as a factor in the maintenance of the diversity of microbial ecosystems. J. Gen. Microbiol., 106, 307-320.

Dopazo, C. P., Lemos, M. L., Loderrios, C., Bolinches, J., Barja, J. L., and Toranzo, A. E. (1988) Inhibitory activity of antibiotic-producing marine bacteria against fish pathogens. J. Appl. Bacteriol., 65, $97-$ 101.

Fredrickson, A. G., and Stephanpoulos, G. (1981) Microbial competition. Science, 213, 972-979.

Fujita, M., Ike, M., and Uesugi, K. (1994) Operation parameters affecting the survival of genetically engineered microorganisms in activated sludge processes. Wat. Res., 28, 1667-1672.

Gauthier, M. J., and Flatas, G. F. (1976) Antibacterial activity of marine violet-pigmented Alteromonas with special reference to the production of brominated compounds. Can. J. Microbiol., 22, 1612-1619.

Gurijala, K. R., and Alexander, M. (1990) Explanation for the decline of bacteria introduced into lake water. Microb. Ecol., 20, 231-244.

Han, K., and Levenspiel, O. (1988) Extended Monod kinetics for substrate, product, and cell inhibition. Biotechnol. Bioeng., 32, 430-437.

Jemsen, L. B., Ramos, J. L., Kaneva, Z., and Molin, S. (1993) A substrate-dependent biological containment system for Pseudomonas putida based on the Escherichia coli gef gene. Appl. Environ. Microbiol., 59, 3713-3717.

Klein, T. M., and Alexander, M. (1986) Bacterial inhibitors in lake water. Appl. Environ. Microbiol., 52, 114-118.

Lemos, M. L., Toranzo, A. E., and Barja, J. L. (1985) Antibiotic activity of epiphytic bacteria isolated from intertidal seaweeds. Microb. Ecol., 11, 149-163.

Lenas, P., and Pavlou, S. (1995) Coexistence of three competing microbial populations in a chemostat with periodically varying dilution rate. Math. Biosci., 129, 111-42.
Lenski, R. E. (1986) Coexistence of two competitors on one resource and one inhibitor: A chemostat model based on bacteria and antibiotics. J. Theor. Biol., 122, 83-93.

Lotka, A. J. (1920). Elements of Physical Biology. Williams and Wilkins, Baltimore.

Nair, S., and Simidu, U. (1987) Distribution and significance of heterotrophic marine bacteria with antibacterial activity. Appl. Environ. Microbiol., 53, 2957-2962.

Ramos, J. L., Diaz, E., Dowling, D., de Lorenzo, V., Molin, S., O 'Gara, F., Ramos, C., and Timmis, K. N. (1994) The behavior of bacteria designed for biodegradation. Bio/Technology, 12, 1349-1356.

Rosenfeld, W. D., and Zobell, C. E. (1947) Anitibiotic production by marine microorganisms. J. Bacteriol., 54, 393-398.

Schoener, T. W. (1976) Alternatives to Lotka-Volterra competition: Models of intermediate complexity. Theor. Pop. Biol., 10, 309-333.

Short, K. A., Doyle, J. D., King, R. J., Seidler, R. J., Stotzky, G., and Olsen, R. H. (1991) Effects of 2,4,dichlorophenol, a metabolite of a genetically engineered bacterium, and 2,4-dichlorophenoxyacetate on some microorganisms-mediated ecological processes in soil. Appl. Environ. Microbiol., 57, 412418.

Snape, J. B., Dunn, I. J., Ingham, J., and Prenosil, J. E. (1995) Dynamics of environmental Bioprocess. Modeling and Simulation. VCH Publishers, New York.

Soda, S., Watatani, H., Ike, M., and Fujita, M. (1998) Factors affecting the survival of exogenous bacteria in microbial ecosystems: Existence of indigenous bacteria with antagonistic activity. Biocontrol Sci., 3, 63-72.

Tang, Y. J., and Alexander, M. (1987) Absence of a role for lytic microorganisms in decline of bacteria and Saccharomyces introduced into soil. Microb. Ecol., 14, 67-73.

Vance, R. R. (1986) The stable coexistence of two competitors for one resource. Am. Nat., 126, 72-86.

Wanner. O., and Gujer, W. (1986) A multispecies biofilm model. Biotechnol. Bioeng., 28, 314-328.

Zaidi, B. R., Murakami, Y., and Alexander, M. (1989) Predation and inhibitors in lake water affect the success of inoculation to enhance biodegradation of organic chemicals. Environ. Sci. Technol., 23, 859863. 